\title{
Characterization of the mTOR pathway in human normal adrenal and adrenocortical tumors
}

\author{
Maria Cristina De Martino, Richard A Feelders, Wouter W de Herder, \\ Peter M van Koetsveld, Fadime Dogan, Joseph A M J L Janssen, A Marlijn Waaijers, \\ Claudia Pivonello', Steven W J Lamberts, Annamaria Colao', Ronald R de Krijger², \\ Rosario Pivonello' and Leo J Hofland
}

Department of Internal Medicine, Division of Endocrinology, Erasmus Medical Center, Rotterdam, The Netherlands 'Dipartimento di Medicina Clinica e Chirurgia, Sezione di Endocrinologia, Università Federico II, Naples, Italy ${ }^{2}$ Department of Pathology, Erasmus Medical Center, Rotterdam and Reinier de Graaf Gasthuis, Delft, The Netherlands

\begin{abstract}
The mTOR pathway has recently been suggested as a new potential target for therapy in adrenocortical carcinomas (ACCS). The aim of the current study is to describe the expression of the mTOR pathway in normal adrenals (NAs) and pathological adrenals and to explore whether there are correlation between the expression of these proteins and the in vitro response to sirolimus. For this purpose, the MTOR, S6K1 (RPS6KB1), and 4EBP1 (EIF4EBP1) mRNA expression were evaluated in ten NAs, ten adrenal hyperplasias (AHs), 17 adrenocortical adenomas (ACAs), and 17 ACCs by qPCR, whereas total(t)/phospho(p)-MTOR, $\mathrm{t} / \mathrm{p}-\mathrm{S} 6 \mathrm{~K}$, and t/p-4EBP1 protein expression were assessed in three NAs, three AHs, six ACAs, and $20 \mathrm{ACCs}$ by immunohistochemistry. The effects of sirolimus on cell survival and/or cortisol secretion in 12 human primary cultures of adrenocortical tumors (ATs) were also evaluated. In NAs and AHs, layer-specific expression of evaluated proteins was observed. S6K1 mRNA levels were lower in ACCs compared with NAs, AHs, and ACAs $(P<0.01)$. A subset of ATs presented a moderate to high staining of the evaluated proteins. Median t-S6K1 protein expression in ACCs was lower than that in ACAs $(P<0.01)$. Moderate to high staining of p-S6K1 and/or p-4EBP1 was observed in most ATs. A subset of ACCs not having moderate to high staining had a higher Weiss score than others $(P<0.029)$. In primary AT cultures, sirolimus significantly reduced cell survival or cortisol secretion only in sporadic cases. In conclusion, these data suggest the presence of an activated mTOR pathway in a subset of ATs and a possible response to sirolimus only in certain ACC cases.
\end{abstract}

Correspondence should be addressed to L J Hofland Email

I.hofland@erasmusmc.nl

\section{Introduction}

Adrenocortical cancer (ACC) is a rare and aggressive cancer with a 5-year survival at the metastatic stage below 15\% (Bilimoria et al. 2008, Baudin et al. 2011, Fassnacht et al. 2011). The insulin-like growth factor (IGF)

\author{
Key Words \\ - adrenal cortex \\ - adenoma \\ - carcinoma \\ - mTOR \\ - immunohistochemistry \\ - adrenocortical cancer \\ - primary cultures
}

system has been considered a major actor in the pathogenesis of ACCs and an attractive target for new treatments in patients affected by this malignancy (Libe \& Bertherat 2005, Almeida et al. 2008, Barlaskar et al. 2009). 
Another important factor may be mTOR, which is a protein kinase of the phosphatidylinositol 3-kinase (PI3K)/Akt signaling pathway, playing a pivotal role in cell growth, metabolism, and proliferation (Guertin \& Sabatini 2007). Activation of the mTOR pathway leads to the phosphorylation and activation of downstream effectors such as the protein 70 ribosomal protein S6 kinase 1 (S6K1) (RPS6KB1) and eukaryotic translation initiation factor 4E-binding proteins (4EBP1) (EIF4EBP1) (Guertin \& Sabatini 2007). S6K1 and 4EBP1 are both regulators of mRNA translation and they stimulate the synthesis of several proteins involved in cell proliferation and survival (Guertin \& Sabatini 2007). Alterations in the mTOR pathway have been found in many human tumors (Wan \& Helman 2007, LoPiccolo et al. 2008, Konings et al. 2009). Therefore, the mTOR pathway is considered a target for antineoplastic therapy in several malignancies and it has recently been proposed as a target for ACC treatment (De Martino et al. 2010, 2012, Doghman et al. 2010).

\section{Materials and methods}

\section{Subjects}

A total of 65 adrenal samples (26 ACCs, 19 adrenocortical adenomas (ACAs), ten adrenal hyperplasias (HAs), and ten normal adrenals (NAs)) were selected from the tissue bank (from 1992 and July 1, 2010) of the Erasmus Medical Centre, Rotterdam (The Netherlands). HAs were obtained from adult patients undergoing bilateral adrenal removal for Cushing's syndrome. All tissues were frozen within 60 min after surgical removal. NAs were obtained from adult donors or adult patients undergoing NA removal during surgery for kidney cancer.

An additional 12 adrenal samples (seven ACCs and five ACAs) were collected during surgery and they were processed immediately to obtain primary adrenal tumor cell cultures.

This study was approved by the Medical Ethics Committee of the Erasmus Medical Center.

The Weiss score, assessed by an expert pathologist in adrenal disease (RRdK), was used to make the distinction between adenomas and carcinomas (Weiss 1984).

The following clinical parameters were recorded in all patients: date of diagnosis, age, gender, ENSAT stage (Fassnacht et al. 2009), Weiss score, mitotic count (as defined by the presence number of mitoses equal or higher than five in 50 high-power fields), hormonal status, and type of hormonal secretion (cortisol and/or androgens and/or estrogens and/or mineralocorticoids) (Fassnacht et al. 2011).

\section{Total RNA isolation and quantitative RT-PCR (qRT-PCR)}

From snap-frozen adrenal tissues, total RNA was isolated using a commercially available kit (High Pure RNA Tissue kit; Roche).

Total RNA from the human ACC cell line NCI-H295R was used as a positive control.

The cDNA synthesis from total RNA and quantitative PCR were performed as described previously (De Martino et al. 2012).

The mRNA expression of MTOR, 4EBP1, S6K1, and the housekeeping gene hypoxanthine phosphoribosyltransferase (HPRT (HPRT1)) were evaluated by quantitative RT-PCR in human adrenocortical tissue samples depending on the availability of frozen tissues.

The primers and probes were purchased from SigmaAldrich. The sequences of the primers and probes used are reported in the Supplementary Table 1, see section on supplementary data given at the end of this article. Samples were normalized against the expression of HPRT. PCR efficiencies (E) were calculated for the primer-probe combinations used (Supplementary Table 1) (Rasmussen 2001). The relative expression of genes was calculated using the comparative threshold method, $2-\Delta \mathrm{Ct}$ (Schmittgen \& Livak 2008), after efficiency correction (Pfaffl 2001) of target and reference gene transcripts (HPRT).

\section{Immunohistochemistry}

The expression of total/phospho-mTOR, total/phospho4EBP1, and total/phospho-S6K1 in adrenal samples were evaluated.

Paraffin-embedded tissue specimens were cut in $5 \mu \mathrm{m}$ sections, deparaffinized, and dehydrated. Antigen retrieval was performed by microwave treatment in Tris-EDTA buffer (pH 9.0). The slides were cooled for $1 \mathrm{~h}$ at $+4{ }^{\circ} \mathrm{C}$ and incubated for $1 \mathrm{~h}$ at room temperature (RT) with the primary antibodies. The primary monoclonal antibodies (MABs) to detect mTOR, phospho-mTOR, 4EBP1, phospho-4EBP1, and phospho-S6K1 were purchased from Cell Signaling Technology (Inc.-3 Trask Lane-Danvers, MA 01923, USA, dilution: $1: 25,1: 50,1: 1200,1: 25$, and 1:100 respectively). The primary MAB to detect S6K1 was purchased from Santa Cruz Biotechnology, Inc. (dilution 1:25). The slides were washed and incubated for $30 \mathrm{~min}$ at RT with secondary antibodies (Poly-AP-Goat anti-Mouse/Rabbit IgG PowerVision +; ImmunoVision Technologies, Duiven, the Netherlands) at the concentration provided by the manufacturer. After washing, staining was visualized by incubation for $30 \mathrm{~min}$ with new fuchsin solution

Published by Bioscientifica Ltd. 
(Pivonello et al. 2004). Only phospho-S6K1 staining was performed and visualized using a Dako Detection System, following a different protocol as described previously (De Martino et al. 2012). All slides were counterstained with hematoxylin and coverslipped. Positive controls included cases of breast cancer with previously proven positivity at immunohistochemistry (IHC) for the protein evaluated. Negative controls included omission of the primary antibody and the incubation with secondary antibodies.

The staining was evaluated independently by two investigators and any discrepancy was resolved by a consensus review. In tumor specimens (ACAs and ACCs), the results were interpreted in a semiquantitative manner using an intensity-proportion scoring system as described previously (Noh et al. 2008). The score was calculated by the sum of the intensity score and the proportion of the stained cells; this provided a score between 0 and 6 . The proportion score was as follows: $0=$ no positivity $($ or $<10 \%) ;+1=<1 / 3$ tumor cell positivity; $+2=1 / 3-2 / 3$ tumor cell positivity; and $+3=$ more than $2 / 3$ tumor cell positivity. The intensity score was as follows: $+1=$ weak staining; $+2=$ intermediate staining; and $+3=$ strong staining. The score 0 was considered as negative, 2-3 as low, $4-5$ as intermediate, and 6 as high. Finally, adrenocortical tumors (ATs) were dichotomously grouped as having intermediate to high expression of the evaluated protein and phosphoproteins (IHC score $\geq 4$ ) or not (IHC score $<4$ ).

In AHs and NAs, we used only the intensity score as indicated above.

To evaluate the correlation between the in vitro response to sirolimus (rapamycin) and the protein expression of the main components of the mTOR pathway, the expression of total/phospho-mTOR, total/ phospho-4EBP1, and total/phospho-S6K1 were evaluated in 12 additional AT samples used to perform AT primary cell cultures. In this additional series, the staining was performed and visualized using a Dako Detection System, following a different protocol as described previously (De Martino et al. 2012). In this system, dilutions of total/phospho-mTOR, total/phospho-4EBP1, and total/ phospho-S6K1 antibodies were 1:50, 1:100, and 1:2400 and 1:50, 1:50, and 1:100 respectively.

\section{Drugs and reagents}

The mTOR inhibitor sirolimus was purchased from LC Laboratories, Inc. (Woburn, MA, USA), dissolved in dimethyl sulfoxide (DMSO) as concentrated $\left(10^{-3} \mathrm{M}\right)$ stock solution (stored at $-20^{\circ} \mathrm{C}$ ), and diluted in $40 \%$ DMSO before use. IGF1 was purchased from Sigma-Aldrich, diluted in $0.01 \mathrm{M}$ acetic acid as concentrated $\left(5 \times 10^{-6} \mathrm{M}\right)$ stock solution (stored at $-20^{\circ} \mathrm{C}$ ), and diluted in culture medium before use.

\section{Cell lines and culture conditions}

The human ACC cell lines H295 and SW13 were obtained from the American Type Culture Collection (Manassas, VA, USA) and from ECACC (Salisbury, Wiltshire, UK) respectively. Cells were cultured, as described previously in detail (De Martino et al. 2012).

\section{Immunocytochemistry: chamber slides}

To evaluate the expression of total/phospho-4EBP1 and total/phospho-S6K1 in ACC cell lines, H295 and SW13 cells were plated on poly-L-lysine-coated culture chamber slides (NUNC A/S, Roskilde, Denmark) for $48 \mathrm{~h}$ in full medium. Subsequently, the medium was refreshed and cells were incubated for $16 \mathrm{~h}$ in a complete medium or starved in serum-free medium containing $0.1 \%$ human serum albumin (medium-HSA), according to the different treatment group assigned. Finally, media were refreshed again and cells were incubated for $30 \mathrm{~min}$ in complete medium or medium containing HSA with or without IGF1 $\left(10^{-8} \mathrm{M}\right)$, according to the different treatment group assigned (Fig. 1). Immunocytochemistry in chamber slides was performed, as described previously in detail (De Martino et al. 2012). The dilutions of total/phosphomTOR, total/phospho-4EBP1, and total/phospho-S6K1 antibodies were 1:2400, 1:50, and 1:100 respectively.

\section{Primary cell culture of human ATs}

Immediately after surgery, the adrenal specimens were minced into small pieces and dissociated with collagenase type I ( $2 \mathrm{mg} / \mathrm{ml}$; Sigma-Aldrich). Cell viability, after Ficoll density gradient separation, was determined by trypan blue exclusion and it always exceeded 90\%. Cells were cultured in DMEM/F12K medium (Invitrogen), supplemented with 5\% FCS, penicillin $\left(1 \times 10^{5} \mathrm{U} / \mathrm{l}\right.$; BristolMeyers Squibb, Woerden, The Netherlands), and L-glutamine ( $2 \mathrm{mmol} / \mathrm{l} ; \mathrm{GIBCO})$. Cells were plated at a density of 100.000 cells per well in 24 well plates (Corning Costar, Schiphol, The Netherlands). After 3-4 days of incubation at $37{ }^{\circ} \mathrm{C}$ in a humidified incubator containing $5 \% \mathrm{CO}_{2}$, the medium was changed and incubations with or without sirolimus were performed for 7 days in quadruplicate. Controls were treated with the vehicle. The medium and test substances were washed every

Published by Bioscientifica Ltd. 


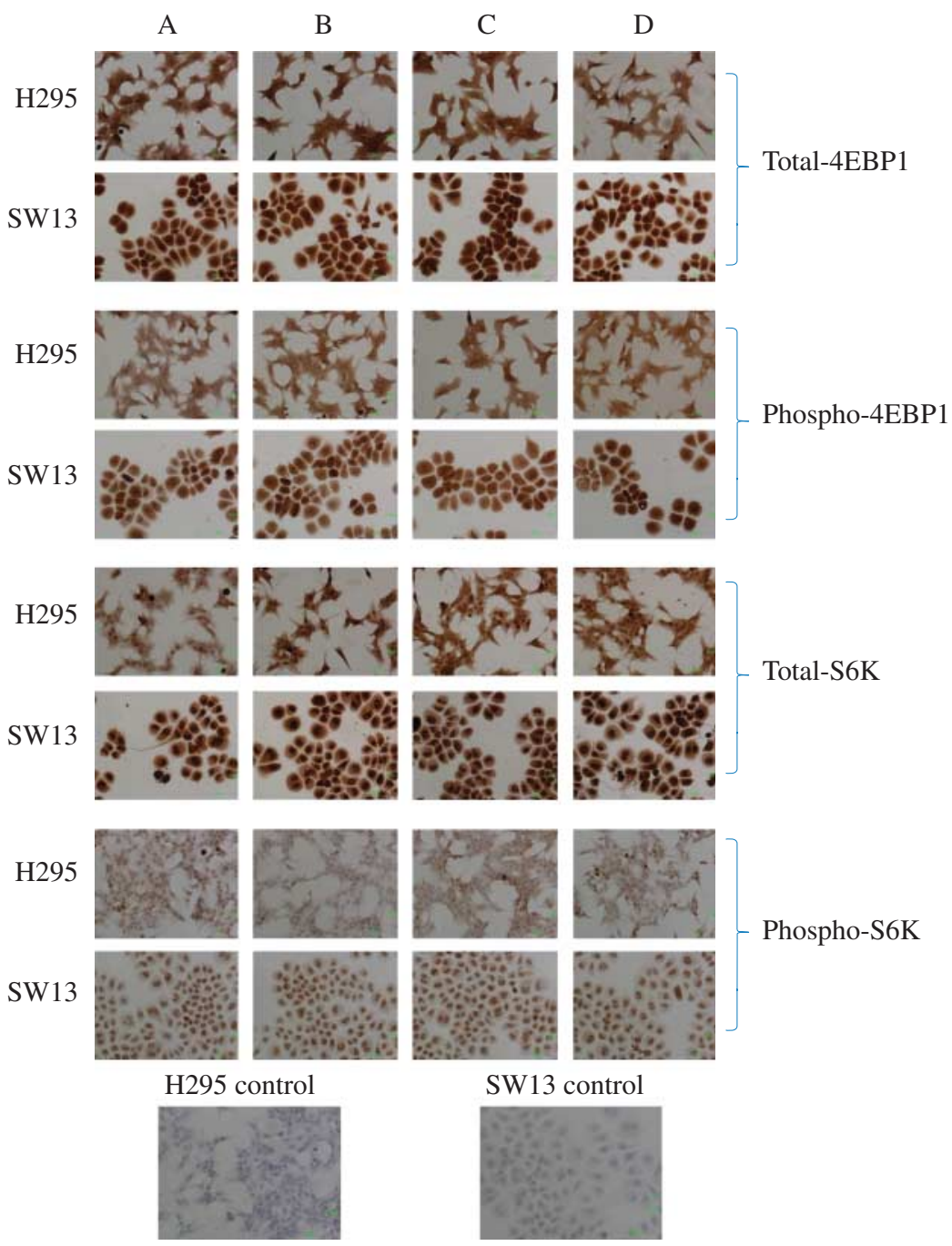

\section{Figure 1}

Immunocytochemical detection of total 4EBP1, phospho-4EBP1, total S6K1, and phospho-S6K1 in two human adrenocortical carcinoma cell lines $\mathrm{H} 295$ and SW13 plated in different medium conditions (details in the text). Panel A medium conditions include a complete continuous medium. Panel $\mathrm{B}$ medium conditions include $48 \mathrm{~h}$ in a complete medium followed by $16 \mathrm{~h}$ in a serum-free medium containing $0.1 \%$ human serum albumin (medium-HSA), thus an additional $30 \mathrm{~min}$ in medium-HSA. Panel $\mathrm{C}$ medium

3 days. On day 7, media were collected, cells were washed twice with saline, followed by lysis for DNA analysis as described previously in detail (Hofland et al. 1990). Owing to a limitation in the cell yield obtained per tissue, and the absence of cortisol secretion in some cases, not all experiments could be carried out in each individual case.

\section{Cortisol secretion assay}

In cortisol-secreting adrenal tumor primary cultures, we evaluated the effects of sirolimus on cortisol production. conditions include $48 \mathrm{~h}$ in a complete medium followed by $16 \mathrm{~h}$ in medium-HSA and thus $30 \mathrm{~min}$ in a complete medium. Panel $\mathrm{D}$ medium conditions include $48 \mathrm{~h}$ in a complete medium followed by $16 \mathrm{~h}$ in medium-HSA and thus $30 \mathrm{~min}$ in medium-HSA with IGF1 $\left(10^{-8} \mathrm{M}\right)$. The two pictures at the bottom show the absence of staining in the negative controls. Magnification, $\times 200$.

The culture supernatants from primary culture experiments, performed as described above, were collected and stored at $-20{ }^{\circ} \mathrm{C}$ until determination of the cortisol concentration.

The cortisol concentration was determined by a nonisotopic, automated chemiluminescence immunoassay system (Siemens Medical Solutions Diagnostics, Breda, The Netherlands). Cortisol levels were expressed as the percentage of control and were corrected for the total DNA content in each well, thereby reflecting cortisol production per cell. 


\section{Statistical analysis}

Statistical analysis was performed using the statistical software of SPSS (SPSS 15.0; SPSS, Inc.). Quantitative data are expressed as means and S.D. and medians and range. Qualitative data are expressed as percentage. The Mann-Whitney $U$ test and the Kruskal-Wallis test were used to compare two or more groups. The comparative statistical evaluations among treatment groups in primary culture experiments were performed by ANOVA, followed by a multiple comparative test (Newman-Keuls). Spearman's rank correlation coefficient was used to test the correlation.

\section{Results}

\section{Study population}

This study included samples from 65 adrenal patients. Only two of the included ACC patients were children (9.5 and 4.2 years old respectively).

To describe the mTOR pathway, the mRNA expression levels of MTOR, 4EBP1, and S6K1 were evaluated by qRTPCR in 54 human adrenocortical tissue samples (17 ACCs, 17 ACAs, ten AHs, and ten NAs) and the protein expression levels of mTOR, phospho-mTOR, 4EBP1, phospho-4EBP1, total S6K1, and phospho-S6K1 were evaluated by IHC in 32 human adrenocortical tissue samples (20 ACCs, six ACAs, three AHs, and three NAs). For 20 cases (11 ACC patients including the two children, and three ACA, three AH, and three NA patients), we had adequate material to perform the analysis of the mTOR pathway by both qRT-PCR and IHC.

\section{mRNA expression of the mTOR pathway components in human adrenal samples}

The mRNA expression of MTOR, 4EBP1, and S6K1 were evaluated by quantitative RT-PCR in 54 adrenocortical tissue samples. In the samples, no statistically significant differences between the expression levels of MTOR (ACCs: 0.16 \pm 0.29 ; ACAs: $0.35 \pm 0.21$; AHs: $0.29 \pm 0.07$; NAs: $0.27 \pm 0.19$, median \pm s.D.) and $4 \mathrm{EBP} 1$ (ACCs: 0.36 \pm 0.60 ; ACAs: 0.41 \pm 0.64 ; AHs: $0.36 \pm 0.42$; and NAs: $0.33 \pm 0.08$ ) were found, while the mRNA expression levels of $S 6 K 1$ were significantly lower in ACCs than in other groups (ACCs: $0.10 \pm 0.08$ vs ACAs: $0.20 \pm 0.11$; AHs: $0.29 \pm 0.07$; and NAs: $0.23 \pm 0.08$; $P<0.01)$ (Fig. 2A, B and C). In the group of ACCs, a significant correlation was found between the mRNA levels of $M T O R$ and $4 E B P 1 \quad(P=0.043)$, MTOR and S6K1 $(P=0.003)$, and $S 6 K 1$ and $4 E B P 1$
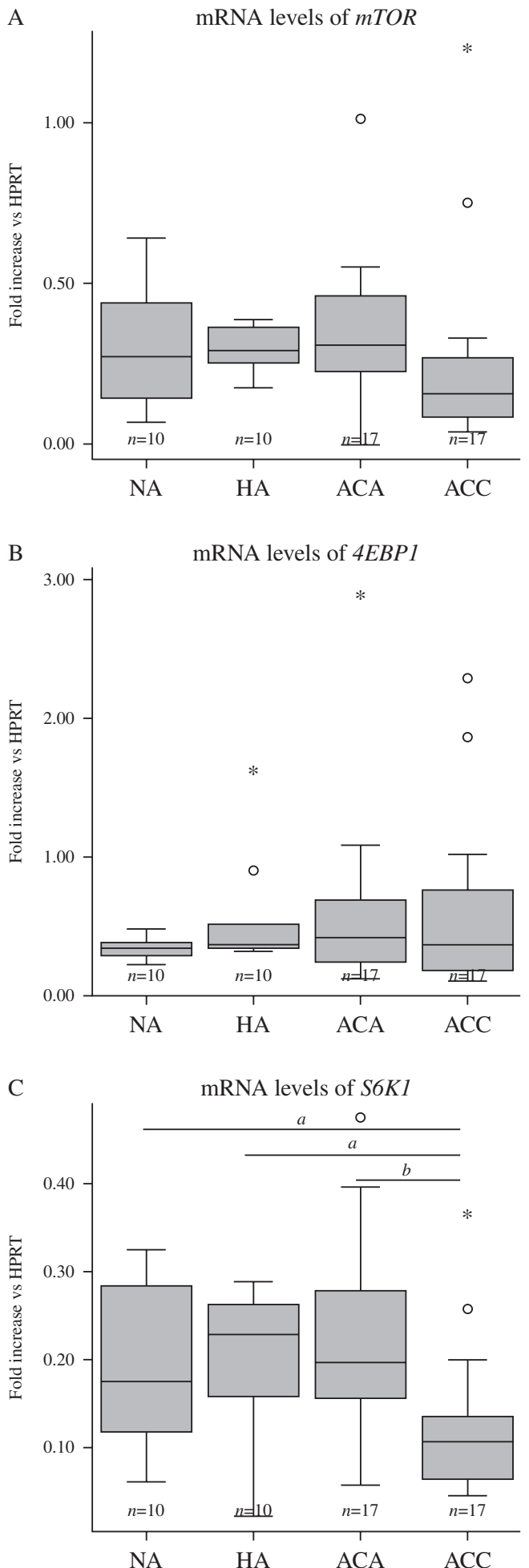

Figure 2

Box plot representation of the relative (A) $m T O R$, (B) 4EBP1, and (C) S6K1 mRNA expression observed in normal and pathological human adrenal samples. A significantly lower $(P<0.01)$ relative mRNA expression of $S 6 K 1$ was observed in adrenocortical carcinomas (ACCs) compared with all the other groups (C). ( ${ }^{\mathrm{a} P}<0.01$; ${ }^{\mathrm{b}} P<0.001$; ${ }^{\circ}$ outliers; * extreme outliers). 
Control

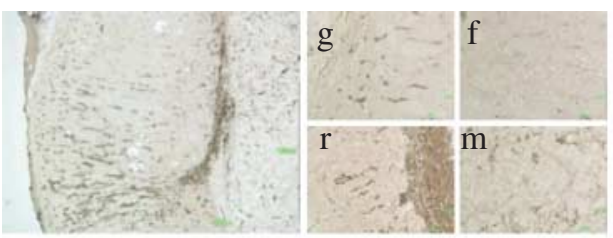

Total-proteins

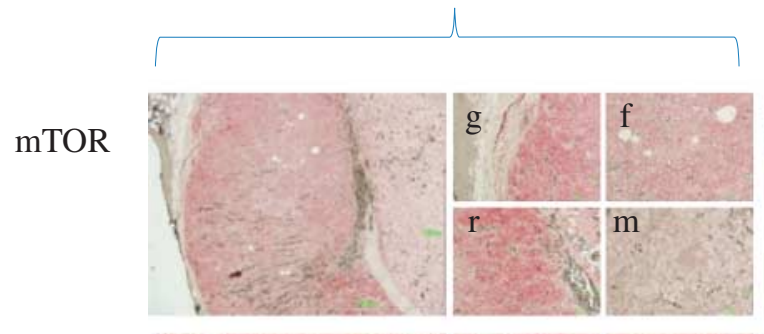

4EBP1
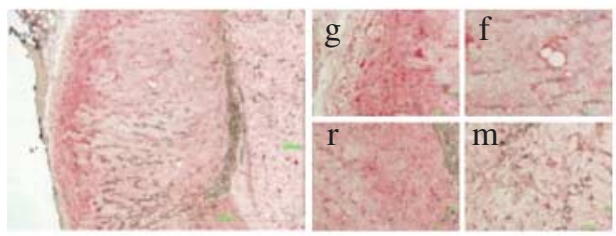

S6K
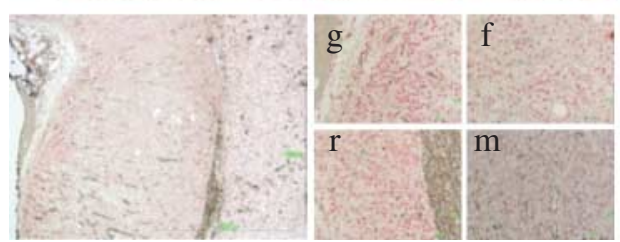

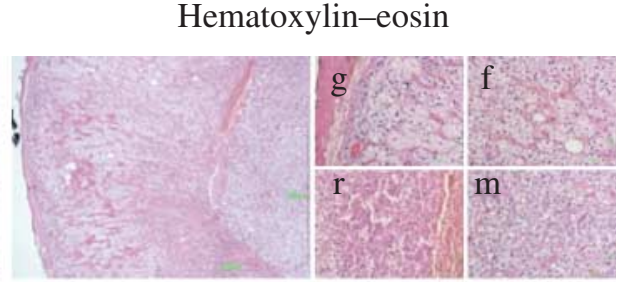

Phospho-proteins
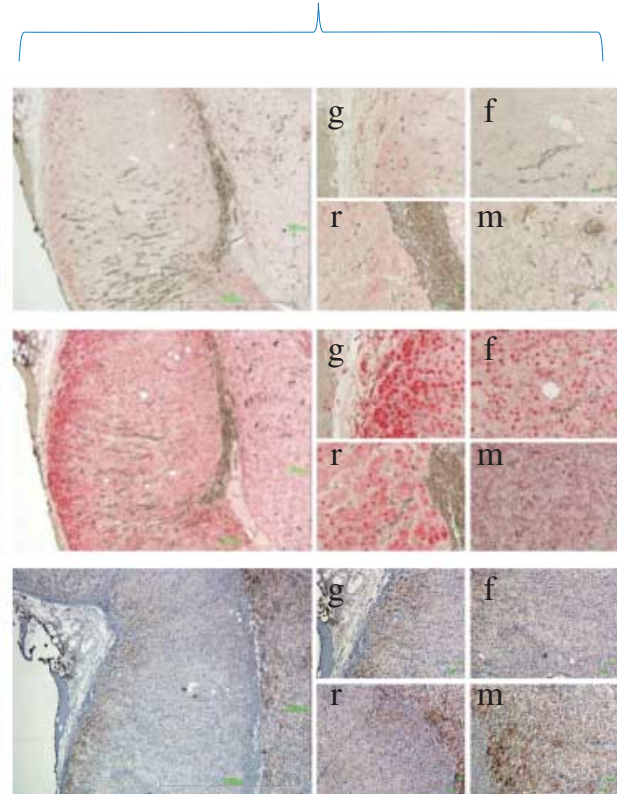

Figure 3

Exemplary pictures of the immunocytochemical detection of total mTOR, phospho-mTOR, total 4EBP1, phospho-4EBP1, total S6K1, and phosphoS6K1 in normal adrenals. Large pictures represent all the normal adrenal

( $P=0.011$, but no relationships were observed with the Weiss score, mitotic index, and TNM. The correlations were also present when a whole series of samples was considered.

\section{Protein expression of the mTOR pathway components in normal human adrenal tumors and $\mathrm{AH}$}

All the evaluated components of the mTOR pathway were expressed in the cortex of the evaluated NA and $\mathrm{AH}$ specimens. However, a layer-specific expression of the evaluated proteins was observed. The intensity of staining for the majority of the evaluated proteins was moderate to strong in glomerulosa and reticularis layers, weak to moderate in fasciculata, and faint to weak in the NA medulla, with the exception of phospho-S6K1, which showed moderate to strong staining in the normal medulla. layers (magnification, $\times 40$ ) and smaller pictures represent the higher magnification $(100 \times)$ of each adrenal layers (from the outer to the inner: glomerulosa (g); fasciculata (f); reticularis $(r)$; and medulla $(m)$ ).

Representative pictures of the staining observed in NAs are reported in Fig. 3.

\section{Protein expression of the mTOR pathway components in human ATs}

In 26 ATs (20 ACCs and six ACAs), the protein expression of the total and phospho-mTOR, total and phospho4EBP1, and total and phospho-S6K1 were evaluated by IHC. Table 1 summarizes the results of the IHC and the main clinical features of the evaluated patients. Figure 4 shows two exemplary cases of immunostaining in ATs (a case of ACC (left panel) and a case of ACA (right panel)).

All ACCs, except one case (19/20; 95\%), and all ACAs evaluated showed a positive staining for total mTOR. This expression was intermediate to high in 12 ACCs (60\%) and in all ACA samples. The staining of phospho-mTOR was

Published by Bioscientifica Ltd 


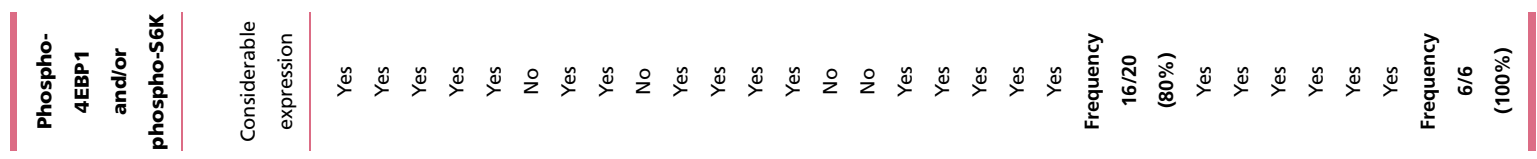

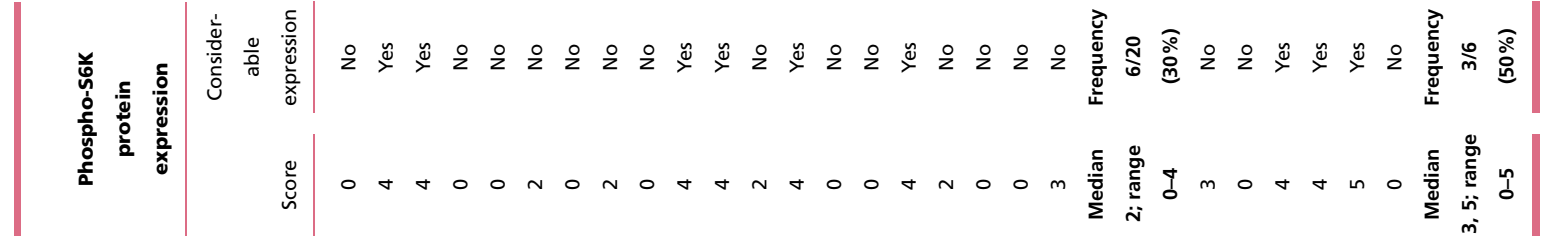

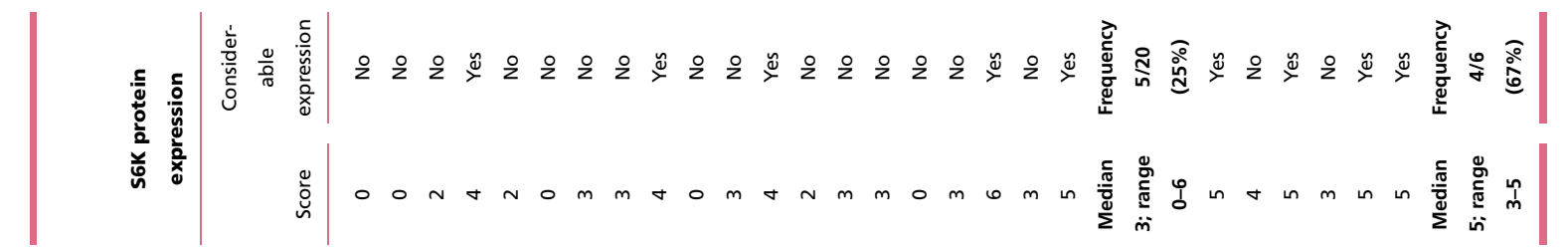

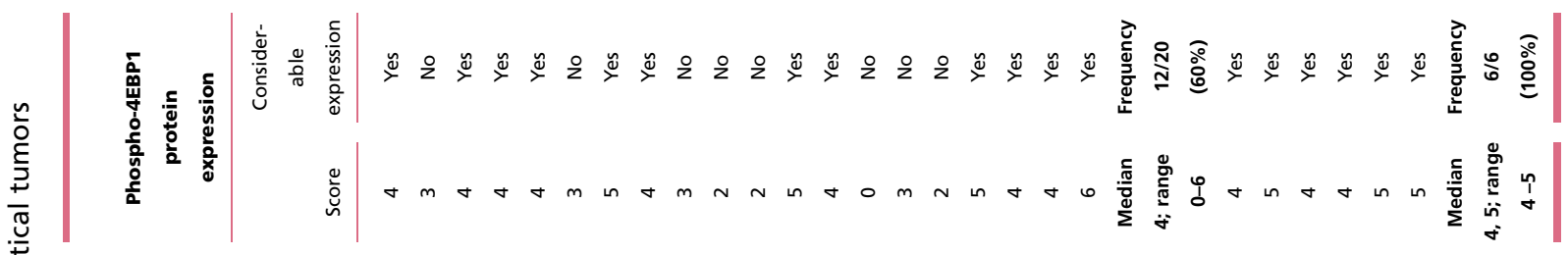

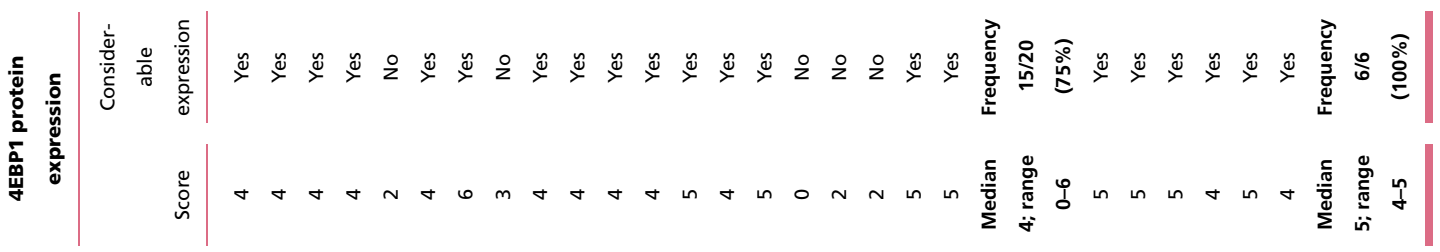

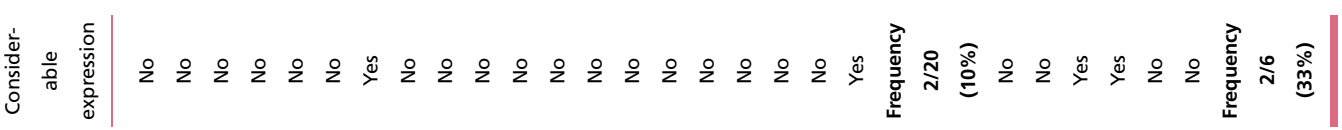

总| n

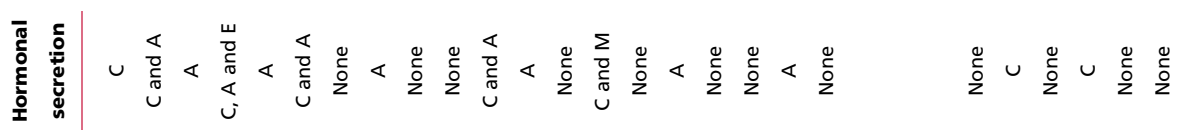

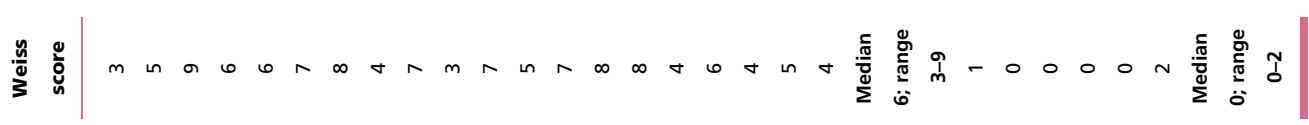

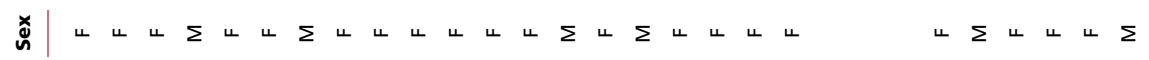

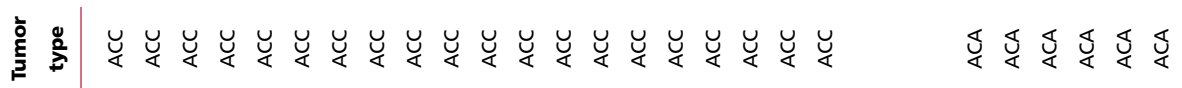

高 I.

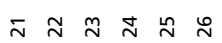




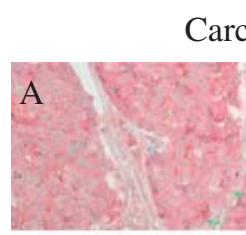

Carcinoma
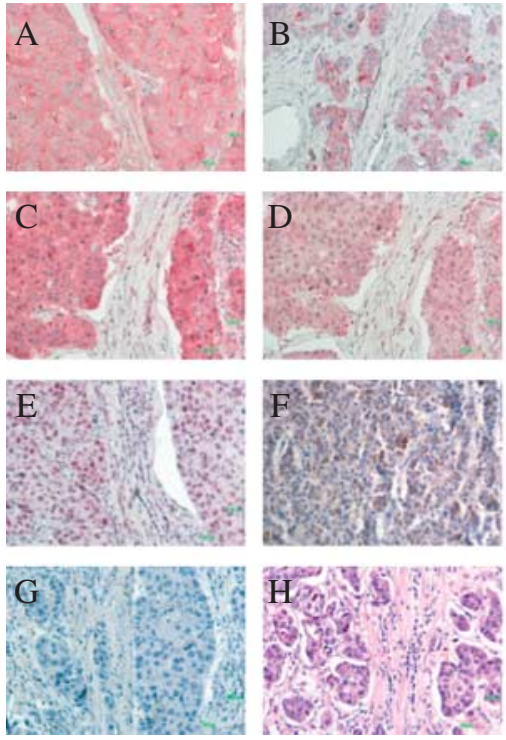

Adenoma
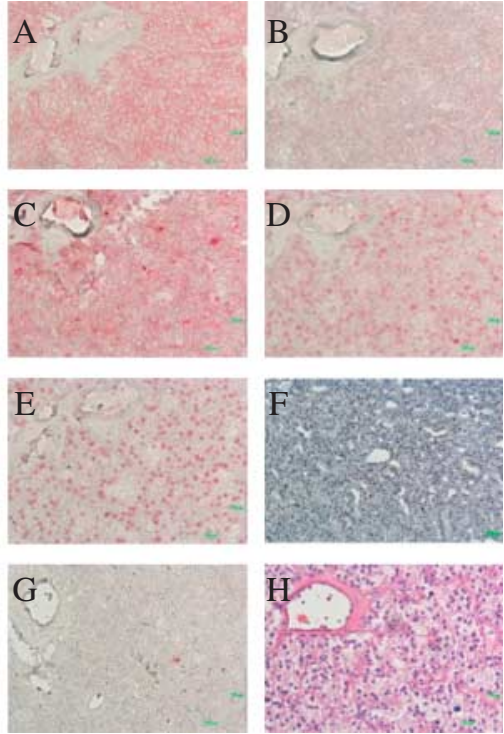

\section{Figure 4}

Immunocytochemical detection of total mTOR (A), phospho-mTOR (B), total 4EBP1 (C), phospho-4EBP1 (D), total S6K1 (E), and phospho-S6K1 (F) in a case of human adrenocortical carcinoma (ACC (left panel)) and a case of

present in a lower number of cases (5/20 ACCs and 3/6 ACAs) and only in some of these it was intermediate to high (two ACCs and two ACAs). A positive staining for total 4EBP1 was observed in all tumor samples evaluated with the exception of one ACC. This staining was intermediate to high in 15 ACCs (75\%) and in all ACAs evaluated. The staining for phospho-4EBP1 was positive in 19 ACCs (95\%) and in all ACAs. This staining was intermediate to high in 12 ACCs (60\%) and in all ACAs. A positive total S6K1 staining was observed in 15 (75\%) ACCs and in all ACAs. This staining was intermediate to high in five ACCs (25\%) and four ACAs (67\%). The staining for phospho-S6K1 was positive in eight ACCs $(40 \%)$ and in four ACAs (67\%). This staining was intermediate to high in six ACCs (30\%) and three ACAs (50\%) respectively. The overall total S6K1 score observed in ACAs was higher than that in ACCs ( $P=0.009)$ (Fig. 5A).

Considering the staining score of phospho-S6K1 and phospho-4EBP1 together, an intermediate to high staining of at least one of the two components of the mTOR pathway was found in 16 ACCs $(80 \%)$ and in all ACAs. An intermediate to high staining of both components was described in two ACCs (10\%) and in three ACAs (50\%). Interestingly, all ATs (six ACAs and 20 ACCs), which did not present any intermediate to high staining of the two above-mentioned phosphoproteins, had significantly higher Weiss scores than others $(P=0.009)$ (Fig. 5B) human adrenocortical adenoma (ACA (right panel)). Picture 'G' shows the absence of staining in the negative controls, and picture ' $\mathrm{H}$ ' the HE staining in both panels. Magnification, $\times 200$.

when considered together. This difference was still present considering only ACCs ( $P=0.029)$ (Fig. 5C).

In ACC group, a higher total S6K1 $(5 \pm 2$ vs $2 \pm 2$; $P=0.04)$ and phospho-4EBP1 ( $5 \pm 1$ vs $3 \pm 2 ; P=0.04$ ) protein expression were observed in tumors having a mitotic count $<5$.

Several positive correlations between the staining score of different components of the mTOR pathway in ATs were observed (Supplementary Table 2, see section on supplementary data given at the end of this article), potentially suggesting common regulators.

In the 14 ATs, for which adequate material was available to perform both IHC and qRT-PCR, none of the evaluated components showed a significant correlation between mRNA and protein expression levels.

ACCs associated with hormonal production did not present any significant difference in the mRNA and protein expression levels of the evaluated components when compared with the non-secreting ACCs.

\section{Protein expression of the mTOR pathway components and in vitro response to sirolimus in preclinical models of human ATs}

In order to explore whether the protein expression of the main components of the mTOR pathway are predictive for the effects of mTOR inhibitors in in vitro models of human

Published by Bioscientifica Ltd. 

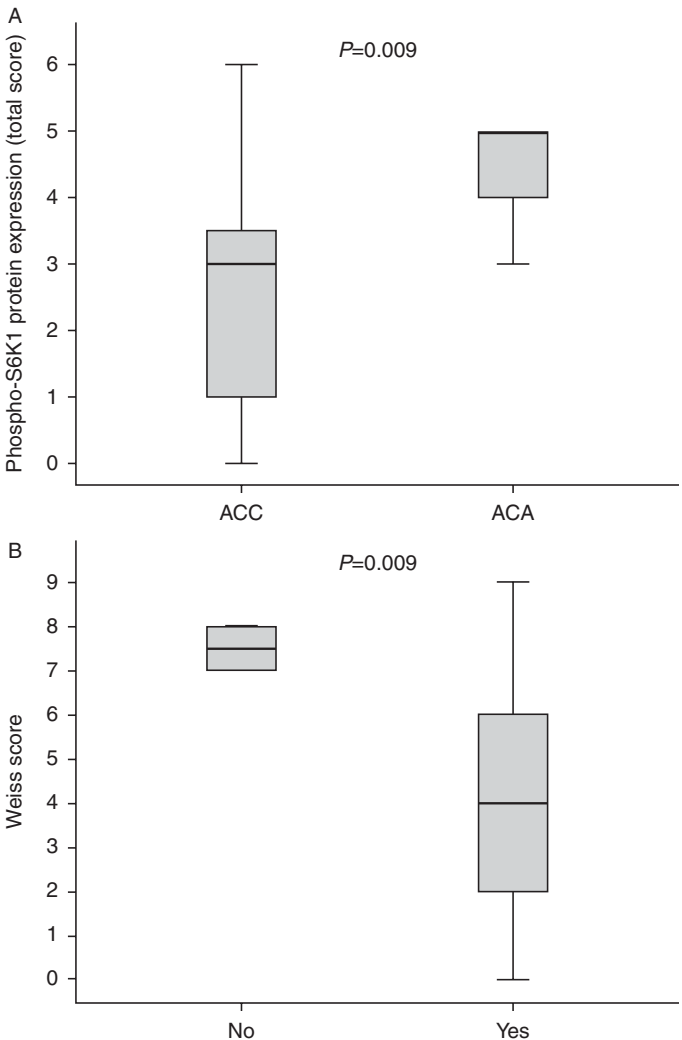

Phospho-4EBP1 and/or phospho S6K-considerable expression

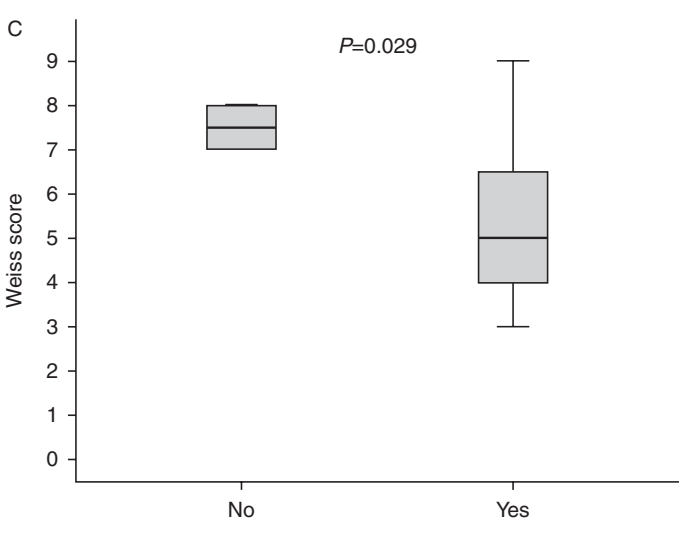

Phospho-4EBP1 and/or phospho S6K-considerable expression

\section{Figure 5}

(A) Box plot representation of total 56K1 protein expression observed in malignant (ACCS) and benign (ACAs) adrenocortical tumors. The expression of S6K1 (total score; explanation in the text) in ACCs was significantly lower than that in ACAs. (B) Box plot representation of the Weiss score values in adrenocortical tumors (including six ACAs and 20 ACCs) divided into two groups according to the presence (yes) or absence (no) of an intermediate to high IHC score for phospho-4EBP1 and/or phospho-S6K1 (explanation in the text). (C) Box plot representation of the Weiss score values in the malignant subgroup of adrenocortical cancer (ACC) divided into two groups according to the presence (yes) or absence (no) of an intermediate to high IHC score for phospho-4EBP1 and/or phospho-S6K1. Tumors not having an intermediate to high IHC score for phospho-4EBP1 and/or phospho-S6K1 had a significantly higher Weiss score than other tumors (B); this difference was still present considering only ACCs (C).
ATs, we performed experiments in human ACC cell lines and primary cell cultures of ACCs and ACAs.

Human ACC cell lines To define whether total/phospho-4EBP1 and total/phospho-S6K1 protein expression are correlated with the in vitro response of ACC cell lines to mTOR inhibitors, immunocytochemistry was performed in $\mathrm{H} 295$ and SW13 cell lines. We had previously described the effects of sirolimus in these cell lines (De Martino et al. 2012) and demonstrated that SW13 cell lines are more sensitive to sirolimus treatment than H295 cell lines. All the evaluated proteins were well expressed in both cell lines, either in basal or in serum/IGF1-stimulated conditions (Fig. 1). No major differences were observed in total/phospho-4EBP1 and total/phospho-S6K1 protein expression between the cell lines.

Primary human ACC and ACA cell cultures To determine whether the protein expression of all evaluated components of the mTOR pathway were correlated with the in vitro response of primary human AT cell cultures to mTOR inhibitors, immunocytochemistry was performed in tissues of seven ACCs and five ACAs from which primary cultures were performed. In these cell cultures, the response to sirolimus $\left(10^{-10}\right.$ and $\left.10^{-6} \mathrm{M}\right)$ was defined based on the presence or absence of significant inhibition in the cell number (as evaluated by DNA analysis) and/or cortisol secretion (in the cortisol-secreting tumors). Supplementary Table 3, see section on supplementary data given at the end of this article shows the results of the effect of sirolimus in primary cultures. Only one ACC primary culture showed a significant cell number reduction after sirolimus treatment (Fig. 6A). In a different case, which was a cortisol-secreting ACC, sirolimus treatment significantly inhibited cortisol secretion (Fig. 6C), but it did not affect the cell number (Fig. 6B). There were no clear differences in the expression of the evaluated proteins between the two responder cases and the others. These results suggest that only a small subset of ACCs show response to mTOR inhibitors and that there is no clear correlation between response to sirolimus and protein expression of the main components of the mTOR pathway.

\section{Discussion}

This study demonstrates a layer-specific protein expression pattern of the major components of the mTOR pathway in NAs and suggests the presence of an activated mTOR pathway in a subset of adrenal tumors.

Published by Bioscientifica Ltd 
A

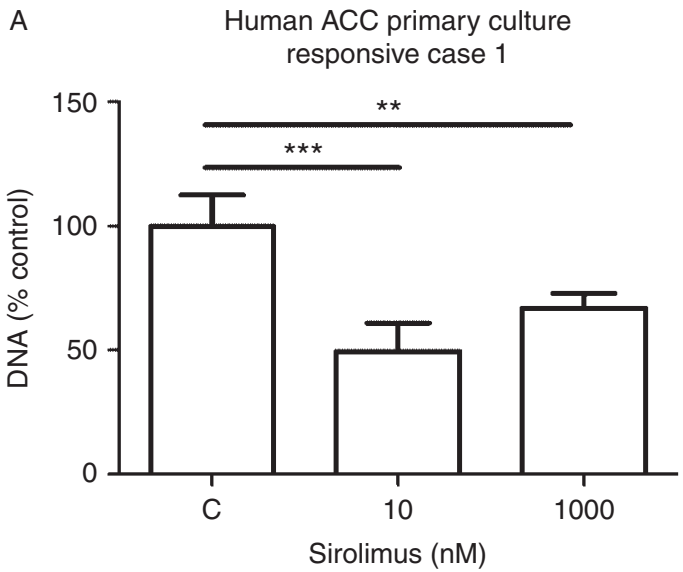

B

Human ACC primary culture responsive case 2

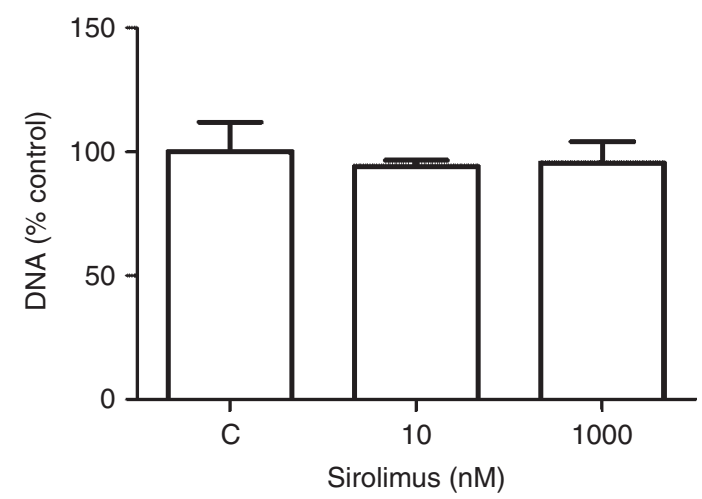

C Human ACC primary culture

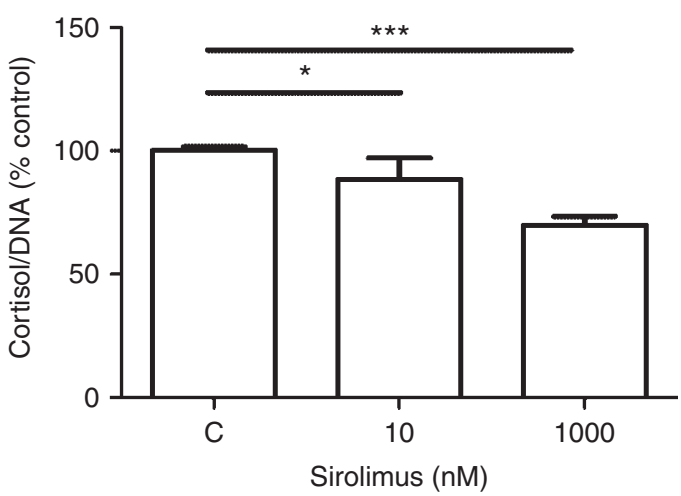

Figure 6

Effects of sirolimus in primary cultures of adrenocortical cancer: two responder cases. (A) A case with a significant reduction in cell survival as measured by the total DNA content. (B and C) A case with a significant reduction in cortisol secretion normalized for the total DNA content (C). Values are expressed as the percentage of the control and are described as mean \pm s.D. of four repeated measurements. ${ }^{*} P<0.05$; ${ }^{*} P<00.01$; $* * * P<0.001$.

http://erc.endocrinology-journals.org DOI: 10.1530/ERC-13-0112
(C) 2014 Society for Endocrinology Printed in Great Britain
Generally, malignant adrenal tumors (ACCs) showed variable protein expression of the evaluated components of the mTOR pathway and lower S6K1 mRNA and protein levels than adrenal benign tumors, suggesting a possible deregulation of the mTOR pathway in ACCs. However, it is difficult to establish whether these downregulations could be related to an abnormal mTOR pathway activity. Phospho-S6K1 and/or phospho-4EBP1 have been considered as potential markers of the mTOR pathway activation in human cancers (Noh et al. 2004, O'Reilly \& McSheehy 2010). Although a higher percentage of benign tumors presented an intermediate to high staining of these phosphoproteins compared with the ACCs, the total scores of phospho-S6K1 or phospho-4EBP1 were not significantly higher in ACAs compared with ACCs. Interestingly, tumors not having intermediate to high staining of these phosphoproteins had a significantly higher Weiss score than the tumors presenting intermediate to high staining of at least one phosphoprotein. These data suggest that the mTOR pathway activity was downregulated in a subgroup of tumors with more aggressive pathological phenotype (although not in all cases with aggressive pathological phenotype), thus indicating that a subset of less differentiated ACCs could be less dependent on the activation of the mTOR pathway.

The different expression of the evaluated proteins in the different layers of the adrenal cortex suggests a specific role of the mTOR pathway in particular adrenal functions. For example, the stronger expression of several components of the mTOR pathway in the reticularis could suggest a role of this pathway in the androgen production and the stronger expression of these components in the glomerulosa may be related to angiotensin II-induced activation of the mTOR pathway (Nazarewicz et al. 2011). We did not perform a real comparison between the staining observed in NAs and that observed in ATs because the presence of different layers in NAs, expressing the evaluated proteins at different intensities, made it difficult to attribute a semiquantitative IHC score as done for tumors. However, if we consider, for example, the most representative layer of the adrenal cortex as reference, which is the fasciculata, some tumors overexpress and others have lower expression of the evaluated proteins. This observation reinforces the suggested heterogeneity of ATs, particularly in ACCs, in the expression of the mTOR pathway.

Several positive correlations between the mRNA levels of the different evaluated components of the mTOR pathway were observed. Similarly, correlations were also observed among the different protein expression levels, suggesting that different components of the mTOR

Published by Bioscientifica Ltd. 
pathway could have common regulators of transcription and/or protein translation. Conversely, we did not find any correlations between the expression observed at mRNA levels and that observed at protein levels. This absence of correlation between protein and mRNA expression could suggest different mechanisms of regulation in protein and mRNA expressions, such as the presence of post-transcriptional regulators, or could simply be explained by the small sample size.

The PI3K/Akt/mTOR pathway is an intracellular pathway that mediates the effects of many growth factors including the IGFs (Liu et al. 2009, De Martino et al. 2010). Preclinical studies demonstrated that mTOR inhibitors inhibit in vitro cell proliferation in ACC cell lines (De Martino et al. 2012) and used a higher dose; they also inhibit the growth of ACC xenografts in immunodeficient mice (Doghman et al. 2010). Moreover, the mTOR pathway has been found to be activated in childhood ACCs (Doghman et al. 2010). However, genomic abnormalities associated with the potential PI3K/Akt/mTOR pathway activation such as the loss of the tumor suppressor gene PTEN or PI3KCA mutations are not common events in ACCs (Ameur et al. 2009, De Martino et al. 2013).

Nakamura et al. (2009), evaluating the presence of potential surrogate markers of targeted drugs in ACCs by IHC, failed to demonstrate significant overexpression of phospho-S6K1 and phospho-4EBP1 in 41 ACCs when compared with 54 ACAs and five NAs. Conversely their results suggest a trend to the downregulation of expression of these proteins in ACCs. This study demonstrates the expression of these proteins for each individual tumor and the percentage of positive/negative cases as established considering only intermediate to high staining. Adopting this approach, we could define different subsets of ACC patients: $10 \%$ with intermediate to high staining of both phospho-S6K1 and phospho-4EBP1; 30 or $60 \%$ expressing phospho-S6K1 or phospho-4EBP1 respectively; and 80\% expressing at least one of these proteins. Based on these results, it can be hypothesized that a subset of patients with ACCs could potentially be a candidate for treatment with mTOR inhibitors. However, it must be considered that tissue biomarkers capable of defining the sensitivity of patients to the mTOR inhibitors have not yet been clearly validated in the clinical setting. To explore the role of the main components of the mTOR pathway as predictors of response in in vitro models of ATs, we performed experiments in human ACC cell lines and primary cell cultures of human ACCs and ACAs. Our previous study had demonstrated that sirolimus inhibits cell proliferation in H295 and SW13 human ACC cell lines although with a different potency, SW13 cell lines being more sensitive than $\mathrm{H} 295$ cell lines (De Martino et al. 2012). These two cell lines express total/phospho-4EBP1 and total/phospho-S6K1 at a comparable level, suggesting that the expression of these proteins is not useful to predict the different sensitivities to sirolimus observed in these cell lines. We further investigated the role of mTOR, phospho-mTOR, 4EBP1, phospho-4EBP1, total S6K1, and phospho-S6K1 as predictors of response to sirolimus treatment in human ACC and ACA cell primary cultures. Also, in this context, protein expression was found to be incapable of predicting a differential sensitivity to sirolimus. In primary culture experiments, sirolimus significantly inhibited total DNA content only in one case of ACC with sarcomatoid features (Coli et al. 2010). These results suggest that only a small subset of ACCs might respond to treatment with mTOR inhibitors. Additionally, they might indicate that ACCs with this particular phenotype could be more sensitive to this treatment. While both ACC cell lines showed a significant response to sirolimus in terms of DNA (De Martino et al. 2012), only one of the seven evaluated ACCs showed a response in this type of experiments. In this regard, it should be considered that primary cultures are in fact unable to proliferate, and therefore, DNA analysis in this context represents cell survival rather than cell proliferation. For this reason, the effects of sirolimus on total DNA content in primary cultures could be underestimated.

IGF2 is known to be overexpressed in ACCs compared with ACAs or non-pathological adrenals, and this explains as to why the IGF pathway has been historically considered as an attractive target for ACCs. The absence of overactivation of the PI3K/Akt/mTOR pathway raises a question as to whether there is any dissociation between the known overexpression of IGF2 in ACCs and the activation of the classical IGF-stimulated intracellular pathway. The variable expression of the evaluated components of the mTOR pathway suggests that mTOR inhibitors must be used with caution in unselected patients with ACCs. Few patients with advanced ACCs who received everolimus, as salvage treatment, did not receive any major benefit (Fraenkel et al. 2013). Few ACC patients have been treated in registered clinical trials (Gangadhar et al. 2011, Quek et al. 2011, Naing et al. 2013). These studies failed to prove the hoped efficacy of these compounds, and they also had limitations. As mitotane can interfere with the metabolism of other drugs including mTOR inhibitors, it became necessary to determine mitotane levels in these patients. However, these early clinical experiences suggest that a subset of patients

Published by Bioscientifica Ltd. 
could benefit (more than 6 months of tumor stabilization) from combined treatment including mTOR inhibitors and IGF1R antagonists (Quek et al. 2011, Naing et al. 2013). In a previous study, we had demonstrated that, in H295 ACC cells, IGFs can activate mechanisms of escape from mTOR inhibitors, which could be responsible for a reduced sensitivity to the treatment with these drugs (De Martino et al. 2012). The blocking of endogenously produced IGF2 increased the antiproliferative effects of sirolimus (De Martino et al. 2012). These preclinical studies support the rational to combine IGF1R antagonist and mTOR inhibitor treatments in a subset of patients with ACCs.

In conclusion, despite the well-known IGF2 overexpression and the potential role of the mTOR pathway in ACCs, presently there is no evidence that clearly supports a key role of this pathway in the pathogenesis of ACCs. However, this study suggests that a subset of ACCs have an activated mTOR pathway. Presently, there is not a strong proven rationale for the use of the mTOR inhibitors alone in ACCs. Therefore, further studies are warranted to investigate the potential role of $\mathrm{mTOR}$ inhibitors, alone or in association with other drugs, in ACC patients. The association between the Weiss score and the expression of the mTOR pathway components should be confirmed in a larger series. Moreover, the role of the expression of the mTOR pathway components as prognostic parameters and as predictive biomarkers for treatment with mTOR inhibitors in ACC warrants further investigation.

\section{Supplementary data}

This is linked to the online version of the paper at http://dx.doi.org/10.1530/ ERC-13-0112.

\section{Declaration of interest}

The authors declare that there is no conflict of interest that could be perceived as prejudicing the impartiality of the research reported.

\section{Funding}

This research did not receive any specific grant from any funding agency in the public, commercial or not-for-profit sector.

\section{Acknowledgements}

The authors thank D M Sprij-Mooij for the expert assistance with qRT-PCR.

\section{References}

Almeida MQ, Fragoso MC, Lotfi CF, Santos MG, Nishi MY, Costa MH, Lerario AM, Maciel CC, Mattos GE, Jorge AA et al. 2008 Expression of insulin-like growth factor-II and its receptor in pediatric and adult adrenocortical tumors. Journal of Clinical Endocrinology and Metabolism 93 3524-3531. (doi:10.1210/jc.2008-0065)

Ameur N, Lacroix L, Motte N, Baudin E, Caillou B, Ducreux M, Elias D, Chanson P, Schlumberger M \& Bidart JM 2009 Mutational status of EGFR, BRAF, PI3KCA and JAK2 genes in endocrine tumors. International Journal of Cancer 124 751-753. (doi:10.1002/ijc.23999)

Barlaskar FM, Spalding AC, Heaton JH, Kuick R, Kim AC, Thomas DG, Giordano TJ, Ben-Josef E \& Hammer GD 2009 Preclinical targeting of the type I insulin-like growth factor receptor in adrenocortical carcinoma. Journal of Clinical Endocrinology and Metabolism 94 204-212. (doi:10.1210/jc.2008-1456)

Baudin E, Leboulleux S, Al Ghuzlan A, Chougnet C, Young J, Deandreis D, Dumont F, Dechamps F, Caramella C, Chanson P et al. 2011 Therapeutic management of advanced adrenocortical carcinoma: what do we know in 2011? Hormones \& Cancer 2 363-371. (doi:10.1007/ s12672-011-0094-2)

Bilimoria KY, Shen WT, Elaraj D, Bentrem DJ, Winchester DJ, Kebebew E \& Sturgeon C 2008 Adrenocortical carcinoma in the United States: treatment utilization and prognostic factors. Cancer 113 3130-3136. (doi:10.1002/cncr.23886)

Coli A, Di Giorgio A, Castri F, Destito C, Marin AW \& Bigotti G 2010 Sarcomatoid carcinoma of the adrenal gland: a case report and review of literature. Pathology, Research and Practice 206 59-65. (doi:10.1016/ j.prp.2009.02.012)

De Martino MC, van Koetsveld PM, Pivonello R \& Hofland LJ 2010 Role of the mTOR pathway in normal and tumoral adrenal cells. Neuroendocrinology 92 (Suppl 1) 28-34. (doi:10.1159/000314280)

De Martino MC, van Koetsveld PM, Feelders RA, Sprij-Mooij D, Waaijers M, Lamberts SW, de Herder WW, Colao A, Pivonello R \& Hofland LJ 2012 The role of mTOR inhibitors in the inhibition of growth and cortisol secretion in human adrenocortical carcinoma cells. Endocrine-Related Cancer 19 351-64. (doi:10.1530/ERC-11-0270)

De Martino MC, Al Ghuzlan A, Aubert S, Assie G, Scoazec JY, Leboulleux S, Do Cao C, Libe R, Nozieres C, Lombes M et al. 2013 Molecular screening for a personalized treatment approach in advanced adrenocortical cancer. Journal of Clinical Endocrinology and Metabolism 98 4080-4088. (doi:10.1210/jc.2013-2165)

Doghman M, El Wakil A, Cardinaud B, Thomas E, Wang J, Zhao W, PeraltaDel Valle MH, Figueiredo BC, Zambetti GP \& Lalli E 2010 Regulation of insulin-like growth factor-mammalian target of rapamycin signaling by microRNA in childhood adrenocortical tumors. Cancer Research 70 4666-4675. (doi:10.1158/0008-5472.CAN-09-3970)

Fassnacht M, Johanssen S, Quinkler M, Bucsky P, Willenberg HS, Beuschlein F, Terzolo M, Mueller HH, Hahner S, Allolio B et al. 2009 Limited prognostic value of the 2004 International Union Against Cancer staging classification for adrenocortical carcinoma: proposal for a Revised TNM Classification. Cancer 115 243-250. (doi:10.1002/ cncr.24030)

Fassnacht M, Libe R, Kroiss M \& Allolio B 2011 Adrenocortical carcinoma: a clinician's update. Nature Reviews. Endocrinology 7 323-335. (doi:10.1038/nrendo.2010.235)

Fraenkel M, Gueorguiev M, Barak D, Salmon A, Grossman AB \& Gross DJ 2013 Everolimus therapy for progressive adrenocortical cancer. Endocrine 44 187-92. (doi:10.1007/s12020-013-9878-1)

Gangadhar TC, Cohen EE, Wu K, Janisch L, Geary D, Kocherginsky M, House LK, Ramirez J, Undevia SD, Maitland ML et al. 2011 Two drug interaction studies of sirolimus in combination with sorafenib or sunitinib in patients with advanced malignancies. Clinical Cancer Research 17 1956-1963. (doi:10.1158/1078-0432.CCR-10-2061)

Guertin DA \& Sabatini DM 2007 Defining the role of mTOR in cancer. Cancer Cell 12 9-22. (doi:10.1016/j.ccr.2007.05.008)

Hofland LJ, van Koetsveld PM \& Lamberts SW 1990 Percoll density gradient centrifugation of rat pituitary tumor cells: a study of functional heterogeneity within and between tumors with respect to growth rates, prolactin production and responsiveness to the somatostatin analog

Published by Bioscientifica Ltd. 
SMS 201-995. European Journal of Cancer 26 37-44. (doi:10.1016/02775379(90)90254-Q)

Konings IR, Verweij J, Wiemer EA \& Sleijfer S 2009 The applicability of mTOR inhibition in solid tumors. Current Cancer Drug Targets 9 439-450. (doi:10.2174/156800909788166556)

Libe RJ \& Bertherat J 2005 Molecular genetics of adrenocortical tumours, from familial to sporadic diseases. European Journal of Endocrinology $\mathbf{1 5 3}$ 477-487. (doi:10.1530/eje.1.02004)

Liu P, Cheng H, Roberts TM \& Zhao JJ 2009 Targeting the phosphoinositide 3-kinase pathway in cancer. Nature Reviews. Drug Discovery 8 627-644. (doi:10.1038/nrd2926)

LoPiccolo J, Blumenthal GM, Bernstein WB \& Dennis PA 2008 Targeting the PI3K/Akt/mTOR pathway: effective combinations and clinical considerations. Drug Resistance Updates 11 32-50. (doi:10.1016/j.drup. 2007.11.003)

Naing A, Lorusso P, Fu S, Hong D, Chen HX, Doyle LA, Phan AT, Habra MA \& Kurzrock R 2013 Insulin growth factor receptor (IGF-1R) antibody cixutumumab combined with the mTOR inhibitor temsirolimus in patients with metastatic adrenocortical carcinoma. British Journal of Cancer 108 826-30. (doi:10.1038/bjc.2013.46)

Nakamura M, Miki Y, Akahira J, Morimoto R, Satoh F, Ishidoya S, Arai Y, Suzuki T, Hayashi Y \& Sasano H 2009 An analysis of potential surrogate markers of target-specific therapy in archival materials of adrenocortical carcinoma. Endocrine Pathology 20 17-23. (doi:10.1007/ s12022-009-9058-2)

Nazarewicz RR, Salazar G, Patrushev N, San Martin A, Hilenski L, Xiong S \& Alexander RW 2011 Early endosomal antigen 1 (EEA1) is an obligate scaffold for angiotensin II-induced, PKC- $\alpha$-dependent Akt activation in endosomes. Journal of Biological Chemistry 286 2886-2895. (doi:10.1074/jbc.M110.141499)

Noh WC, Mondesire WH, Peng J, Jian W, Zhang H, Dong J, Mills GB, Hung MC \& Meric-Bernstam F 2004 Determinants of rapamycin sensitivity in breast cancer cells. Clinical Cancer Research 10 1013-1023. (doi:10.1158/1078-0432.CCR-03-0043)
Noh WC, Kim YH, Kim MS, Koh JS, Kim HA, Moon NM \& Paik NS 2008 Activation of the mTOR signaling pathway in breast cancer and its correlation with the clinicopathologic variables. Breast Cancer Research and Treatment 110 477-483. (doi:10.1007/s10549-007-9746-x)

O'Reilly T \& McSheehy PM 2010 Biomarker development for the clinical activity of the mTOR inhibitor everolimus (RAD001): processes, limitations, and further proposals. Translational Oncology 3 65-79. (doi:10.1593/tlo.09277)

Pfaffl MW 2001 A new mathematical model for relative quantification in real-time RT-PCR. Nucleic Acids Research 29 e45. (doi:10.1093/nar/ 29.9.e45)

Pivonello R, Ferone D, de Herder WW, de Krijger RR, Waaijers M, Mooij DM, van Koetsveld PM, Barreca A, De Caro ML, Lombardi G et al. 2004 Dopamine receptor expression and function in human normal adrenal gland and adrenal tumors. Journal of Clinical Endocrinology and Metabolism 89 4493-4502. (doi:10.1210/jc.2003031746)

Quek R, Wang Q, Morgan JA, Shapiro GI, Butrynski JE, Ramaiya N, Huftalen T, Jederlinic N, Manola J, Wagner AJ et al. 2011 Combination mTOR and IGF-1R inhibition: phase I trial of everolimus and figitumumab in patients with advanced sarcomas and other solid tumors. Clinical Cancer Research 17 871-879. (doi:10.1158/1078-0432. CCR-10-2621)

Rasmussen R 2001 Quantification on the LightCycler. In Rapid Cycle Realtime PCR, Methods and Applications, pp 21-34. Eds S Meuer, C Wittwer \& K Nagakawara. Heidelberg: Springer Press.

Schmittgen TD \& Livak KJ 2008 Analyzing real-time PCR data by the comparative C(T) method. Nature Protocols 3 1101-1108. (doi:10.1038/nprot.2008.73)

Wan X \& Helman LJ 2007 The biology behind mTOR inhibition in sarcoma. Oncologist 12 1007-1018. (doi:10.1634/theoncologist.12-8-1007)

Weiss LM 1984 Comparative histologic study of 43 metastasizing and nonmetastasizing adrenocortical tumors. American Journal of Surgical Pathology 8 163-169. (doi:10.1097/00000478-198403000-00001)

Received in final form 9 May 2014

Accepted 27 May 2014

Made available online as an Accepted Preprint

2 June 2014 http://erc.endocrinology-journals.org DOI: 10.1530/ERC-13-0112
(C) 2014 Society for Endocrinology Printed in Great Britain
Published by Bioscientifica Ltd. 